\title{
A multicenter, double-blind, randomized, comparison study of the efficacy and safety of tigecycline to imipenem/cilastatin to treat complicated intra-abdominal infections in hospitalized subjects in China
}

This article was published in the following Dove Press journal:

Therapeutics and Clinical Risk Management

\author{
Yijian Chen, ${ }^{1,2, *}$ Demei \\ Zhu, ${ }^{1,2, *}$ Yingyuan Zhang, ${ }^{1,2}$ \\ Yongjie Zhao, ${ }^{3}$ Gang Chen, ${ }^{4}$ \\ Ping Li, ${ }^{5}$ Lihong $\mathrm{Xu},{ }^{6}$ Ping \\ Yan, ${ }^{6}$ M Anne Hickman, ${ }^{7}$ \\ Xiajun Xu, ${ }^{6}$ Margaret \\ Tawadrous, ${ }^{7}$ Michele Wible ${ }^{7}$ \\ 'Institute of Antibiotics, Huashan \\ Hospital Fudan University, Shanghai, \\ China; ${ }^{2}$ Key Laboratory of Clinical \\ Pharmacology of Antibiotics, \\ National Health and Family Planning \\ Commission, Shanghai, China; \\ ${ }^{3}$ Department of General Surgery, \\ Tianjin Union Medical Center, \\ Tianjin, China; ${ }^{4}$ Department of \\ Hepatobiliary Surgery, First People's \\ Hospital of Kunming, Kunming, \\ China; ${ }^{5}$ Department of General \\ Surgery, Sichuan Provincial People's \\ Hospital, Chengdu, China; ${ }^{6}$ Pfizer \\ (China) Research \& Development \\ Co, Ltd, Shanghai, China; ' $P$ Pfizer Inc., \\ Collegeville, PA, USA \\ *These authors contributed equally \\ to this work
}

Correspondence: Yingyuan Zhang Institute of Antibiotics, Huashan Hospital, Fudan University, 12 Wulumuqi Zhong Road, Shanghai 200040, China

Tel +86 2l 52888191

Fax +86 21 62488290

Email yyzhang39@hotmail.com
Purpose: To assess the efficacy and safety of tigecycline in treating complicated intra-abdominal infections (cIAIs) in hospitalized patients in China.

Patients and methods: A Phase IV, multicenter, randomized, double-blinded, activecontrolled, non-inferiority study was conducted. Hospitalized cIAI patients $\geq 18$ years of age were randomized (1:1) to receive intravenous tigecycline (initial dose $100 \mathrm{mg}$, then $50 \mathrm{mg} \mathrm{q12h}$ ) or imipenem/cilastatin (500 mg/500 mg or adjusted for renal dysfunction, q6h) for 5-14 days. The primary end point was clinical response for clinically evaluable (CE) subjects at test-ofcure (TOC) assessment.

Results: Four hundred and seventy subjects were randomized; 232 in the tigecycline and 231 in the imipenem/cilastatin group were treated. Tigecycline was non-inferior to imipenem/ cilastatin with respect to clinical response at TOC for all CE subjects, ie, the lower bound of the two-sided $95 \%$ CI $(-12.0 \%,-1.4 \%)$ for the treatment difference in cure rate, tigecycline $(89.9 \%)$ minus imipenem/cilastatin $(96.6 \%)$, was $>-15 \%$. As non-inferiority was concluded in the CE population, superiority of tigecycline over imipenem/cilastatin and superiority of imipenem/cilastatin over tigecycline were tested on the $\mathrm{CE}$ and the modified intent-to-treat (mITT) populations according to pre-specified statistical criteria, and neither could be demonstrated (the cure rate was $82.8 \%$ vs $88.7 \%$, difference $-6.0 \%$ [ $-12.8 \%, 0.8 \%]$, for the mITT population). The subject-level microbiological response rate at TOC for the microbiologically evaluable population was $88.0 \%(110 / 125)$ vs $95.3 \%(102 / 107$, difference $-7.3 \%[-15.2 \%$, $0.5 \%]$ ). Nausea, drug ineffectiveness, postoperative wound infection, vomiting, and pyrexia were the most common adverse events in tigecycline-treated subjects; pyrexia, nausea, vomiting, and increased alanine aminotransferase and aspartate aminotransferase levels were most common in imipenem/cilastatin-treated subjects; none were unanticipated.

Conclusion: Tigecycline was non-inferior to imipenem/cilastatin in treating hospitalized adult patients with cIAI. Superiority of tigecycline over imipenem/cilastatin or imipenem/cilastatin over tigecycline could not be demonstrated. Safety was consistent with the known profile for tigecycline.

ClinicalTrials.gov identifier: NCT01721408.

Keywords: tigecycline, imipenem/cilastatin, complicated intra-abdominal infections, noninferiority 


\section{Introduction}

Complicated intra-abdominal infections (cIAIs) are common health problems including a wide range of pathological conditions from appendicitis to peritonitis with a mortality rate of $10.5 \%{ }^{1,2}$ Aerobic and anaerobic bacteria are often found concurrently in cIAI. ${ }^{3,4}$ In southern China, the causes of cIAI include Escherichia coli (47.6\%), Klebsiella pneumoniae (16.9\%), Enterococcus faecalis (10.4\%), and Pseudomonas aeruginosa $(8.8 \%){ }^{5}$ The highest levels of antimicrobial resistance among Gram-negative bacilli causing IAIs have been found in Asia-Pacific countries where resistance is increasing. ${ }^{6-9}$ Growing antimicrobial resistance has led to increased difficulty in treating cIAI.

Tigecycline is a glycylcycline antibiotic that inhibits the growth of many resistant Gram-negative aerobic and anaerobic bacteria, as well as Gram-positive bacteria (including vancomycin-resistant Enterococci and methicillinresistant Staphylococcus aureus). ${ }^{10}$ Tigecycline has demonstrated efficacy in treating patients with cIAIs and was non-inferior to comparators in randomized, multicenter, double-blind, or open-label Phase III and Phase IV comparative trials. ${ }^{11-13}$ In Chinese patients with cIAI, tigecycline monotherapy was also effective and demonstrated a consistent safety profile. ${ }^{14}$

Tigecycline was approved for the indication of cIAI by the China Food and Drug Administration in $2010 .{ }^{15}$ A postapproval study to observe the efficacy and safety of tigecycline as a viable new therapy for cIAIs in a larger population would provide valuable information, hence the current study. Considering that imipenem/cilastatin is a highly effective monotherapeutic agent with a wide spectrum of activity that is approved for the treatment of IAIs in China, it was chosen as the comparative agent. This post-approval, randomized, double-blinded, active-controlled, non-inferiority study (ClinicalTrials.gov: NCT01721408) evaluated the efficacy of tigecycline compared with imipenem/cilastatin in treating a larger population of hospitalized subjects with cIAI in China. In addition, this study gathered in vitro susceptibility data on tigecycline for a range of bacteria causing cIAI, compared microbiological efficacy between treatment groups, and determined the safety of tigecycline compared with the imipenem/cilastatin in this patient population.

\section{Patients and methods}

\section{Ethics}

The study was conducted at 47 investigational centers in China between November 2012 and October 2015 and was approved by each study center's Independent Ethics
Committee and was conducted in compliance with the Declaration of Helsinki and International Conference on Harmonization Good Clinical Practice Guidelines. All local regulatory requirements were followed. A signed and dated informed consent was obtained before screening.

\section{Study design}

This was a Phase IV, multicenter, randomized, double-blind (ie, subject-, investigator-, and sponsor-blinded) study. Patients $\geq 18$ years of age who met the entry criteria for cIAI were randomized (the participating study center contacted the interactive response technology [eg, interactive voice response or interactive web response] provider to randomize the eligible subject into the study) in a $1: 1$ ratio to receive intravenous (IV) tigecycline or IV imipenem/cilastatin. The randomization was stratified by Acute Physiology and Chronic Health Evaluation II score $(\leq 15$ vs $>15)$. The treatment duration was 5-14 days, and the test-of-cure (TOC) assessment was performed between 14 and 21 days after the last day of therapy (end of treatment, [EOT]). The primary end point was clinical response within the clinically evaluable (CE) population at TOC. Use of systemic antibacterial agents to which the causative pathogen of intra-abdominal infection was susceptible, intra-abdominal topical use of antibacterial agents, and transition to oral antibiotic therapy were not permitted. Other anti-infective drugs such as antifungal agents, acyclovir, oral vancomycin (for treatment of Clostridium difficile), and ophthalmic aminoglycosides were permitted, as required.

Unblinded safety data were summarized and reviewed by an external Data Monitoring Committee (DMC) on a periodic basis. The sponsor designated a biostatistician not affiliated with the project to prepare unblinded safety data for periodic DMC review. The DMC was empowered to recommend modifications to the protocol to enhance subject safety or early termination if there was a significant safety hazard. DMC recommendations were forwarded to the sponsor for final decision.

\section{Inclusion and exclusion criteria}

Subjects must meet all of the following inclusion criteria to be eligible for enrollment into the study. The inclusion criteria included: 1 ) hospitalized male or female subjects $\geq 18$ years of age; 2) laparotomy, laparoscopy, or percutaneous drainage of an intra-abdominal abscess within 24 hours of enrollment; 3) for subjects enrolled preoperatively, study drugs were given with strong suspicion or confirmed diagnosis of IAI with baseline intra-abdominal culture taken from the 
infected site; 4) cIAI duration $<2$ weeks; 5) confirmation of minimal clinical criteria when IAI was diagnosed or highly suspected; and 6) agreement to use a highly effective contraceptive for females of childbearing potential.

Patients were excluded if they met any of the following criteria: 1) had any concomitant condition that could affect the study treatment and evaluation; 2) had leukemia or systemic malignancy requiring therapy within 3 months before study entry, or any metastatic malignancy to the abdomen with life expectancy $<6$ months; 3 ) had an anticipated length of antibiotic therapy $<5$ days or were unlikely to complete the course of treatment; 4) received concomitant treatment with ganciclovir; 5) had hepatic/renal/bone marrow function failure; 6) had IAI caused by organism(s) not susceptible to study drugs; 7) received $>24$ hours of non-study systemic antibiotics within 72 hours before enrollment without treatment failure noted; or 8) had infection requiring systemic antimicrobial therapy at a site other than abdomen.

For additional details, see Table S1.

\section{Treatments}

Tigecycline was administered with an initial IV dose of $100 \mathrm{mg}$ followed by $50 \mathrm{mg}$ twice a day, approximately every 12 hours. For blinding purposes, placebo (normal saline $100 \mathrm{~mL}$ IV) was administered every 12 hours beginning 6 hours after the initial IV dose of tigecycline. Patients in the imipenem/cilastatin group received treatment approximately every 6 hours intravenously. For patients with creatinine clearance $\left(\mathrm{Cl}_{\mathrm{CR}}\right) \geq 71 \mathrm{~mL} / \mathrm{min} / 1.73 \mathrm{~m}^{2}$, imipenem/cilastatin $500 \mathrm{mg} / 500 \mathrm{mg}$ was given; for those with estimated $\mathrm{Cl}_{\mathrm{CR}}<71 \mathrm{~mL} / \mathrm{min} / 1.73 \mathrm{~m}^{2}$, the dosage was adjusted according to the patient's baseline weight and estimated clearance. From EOT to TOC, any non-study antibacterial treatments were prohibited.

\section{Efficacy evaluations}

Clinical response was classified as: cure, failure, or indeterminate. Microbiological response was evaluated at the subject level and pathogen level as eradication, persistence, indeterminate, or superinfection (subject level only) (Table 1).

Patients were evaluated daily during the treatment period and at the TOC visit. Clinical response to therapy was recorded on Day 3, at EOT, and at TOC assessment. The primary efficacy end point was clinical response in the CE population at the TOC assessment. The secondary end points were clinical response at TOC and microbiological response at the subject level at TOC in the microbiologically evaluable (ME) population. Additional efficacy end points included: clinical response and microbiological response at the subject level at the EOT assessment and by monomicrobial and polymicrobial infections (ie, infected by 2,3 , or $\geq 4$ pathogens) at both the TOC and EOT assessments, clinical response by baseline isolate, microbiological response at the pathogen level, clinical response and microbiologic response by minimum inhibitory concentration (MIC) value for each baseline isolate, susceptibility data $\left(\mathrm{MIC}_{50}\right.$ and $\left.\mathrm{MIC}_{90}\right)$ for each baseline isolate, and development of decreased susceptibility ( $\geq 4$-fold increase in MIC).

\section{Safety evaluations}

Safety evaluations included adverse events (AEs), vital signs, safety laboratory tests, and physical examinations. Worsened clinical signs and symptoms of the infection were recorded as AEs. Serious AEs (SAEs) included death, life-threatening conditions, hospitalization or prolonged hospitalization, persistent or significant disability/incapacity, or congenital anomaly/birth defect. Lack of efficacy was considered as an SAE and coded as "drug ineffective".

\section{Microbiological determinations}

Two sets of blood cultures were obtained at two different body sites within 24 hours before administrating the first dose of study drug (Day 1).

Bacterial cultures (aerobic and anaerobic) from the IAI site were analyzed at baseline, and, when performed, during and after treatment. Microbiology testing was performed by a local laboratory at each study center and retested by a central laboratory (Huashan Hospital, Fudan University, China). The established breakpoints for tigecycline were used. ${ }^{16}$

\section{Statistical methods}

Determination of sample size

Assuming that tigecycline and imipenem/cilastatin were equally effective, with favorable clinical response rates (ie, cure rates) of $75 \%$ at the TOC assessment, 175 subjects per treatment group were required to ensure $90 \%$ probability that the lower limit of a two-sided 95\% CI (based on the normal approximation) for the true difference (tigecycline minus imipenem/cilastatin) in cure rate was greater than $-15 \%$. Assuming a clinical evaluability rate $\geq 75 \%, \sim 470$ subjects were required to be enrolled to obtain $350 \mathrm{CE}$ subjects.

\section{Analysis populations and analysis sets}

The modified intent-to-treat (mITT) population included all randomized subjects (ie, the ITT population) who received $\geq 1$ dose of study drug. The CE population comprised all mITT 
Table I Definitions of clinical response and microbiological response

\begin{tabular}{|c|c|}
\hline & Definition \\
\hline \multicolumn{2}{|c|}{ Clinical response } \\
\hline Cure & $\begin{array}{l}\text { The study drug (ie, tigecycline or imipenem/cilastatin) and the initial intervention (operative and/or radiologically } \\
\text { controlled drainage procedure) resolved the intra-abdominal infection. The relevant clinical signs and symptoms of } \\
\text { infection at baseline disappeared or recovered to normal, and relevant non-microbiological results of laboratory } \\
\text { tests returned to normal level (radiological test was at the discretion of investigators) or the resolution of signs and } \\
\text { symptoms so that no further therapy was required. } \\
\text { If the subject underwent a percutaneous drainage at baseline, did not respond to treatment within } 72 \text { hours of the } \\
\text { initial drainage, and had to undergo an operation and then improved that he/she was considered a clinical cure, the } \\
\text { reason for the failed percutaneous drainage was to be recorded, eg, bowel perforation. The subject must not have } \\
\text { received additional antibacterial agents during treatment. } \\
\text { During the completion of study drug (from the EOT to TOC), any non-study anti-infection products or treatments } \\
\text { were prohibited. }\end{array}$ \\
\hline Failure & $\begin{array}{l}\text { The subject required additional surgical or radiologic intervention and/or received additional anti-infection therapy to } \\
\text { cure the infection (included surgical wound infections) since administration of study drug until TOC; or death after } \\
\text { study Day } 2 \text { due to the infection or a treatment-related AE or discontinuation from investigational product due to a } \\
\text { treatment-related AE (as primary reason) or received greater than } 120 \% \text { of the prescribed number of investigational } \\
\text { product doses. } \\
\text { Subjects could have been declared a therapeutic failure after receiving at least } 2 \text { days ( } 8 \text { doses) of investigational } \\
\text { product. Patients receiving oral switch therapy were considered clinical failures. If a subject was a clinical failure } \\
\text { while receiving the study drug, the subject's clinical response of failure was to be carried forward through the TOC } \\
\text { visit (regardless if they were cured on other antibiotics). Subjects who were clinical failures were to have the TOC } \\
\text { assessment performed prior to the initiation of the non-study antibiotic therapy. }\end{array}$ \\
\hline Indeterminate & $\begin{array}{l}\text { Subjects who were lost to follow-up (failure to have an outcome determination); or who died within } 2 \text { days after the } \\
\text { first dose of the study drug for any reason; or who died after study Day } 2 \text { but prior to the TOC assessment because } \\
\text { of non-infection-related reasons (as judged by the investigator). }\end{array}$ \\
\hline
\end{tabular}

\begin{tabular}{l|l}
\hline Microbiological response at the baseline pathogen level \\
$\begin{array}{l}\text { Eradication (documented or } \\
\text { presumed) }\end{array}$ & $\begin{array}{l}\text { The baseline pathogen was absent in repeat cultures obtained from the original site of the intra-abdominal infection } \\
\text { through the TOC assessment; or a clinical response of cure precluded the necessity of a repeat intra-abdominal } \\
\text { culture. }\end{array}$ \\
\hline $\begin{array}{l}\text { Persistence (documented or } \\
\text { presumed) }\end{array}$ & $\begin{array}{l}\text { Baseline pathogen was present in repeat cultures obtained from the original site of the intra-abdominal abscess, } \\
\text { peritonitis, or surgical wound infection during the study, or clinical outcomes were failure in subjects from whom no } \\
\text { repeat microbiological data was available. }\end{array}$ \\
\hline Indeterminate & $\begin{array}{l}\text { Subjects who were lost to follow-up (did not have an outcome determination); or who died within 2 days after } \\
\text { the first dose of investigational product for any reason; or who died after 2 days but prior to the TOC assessment } \\
\text { because of non-infection-related reasons (as judged by the investigator); or no baseline pathogens identified. }\end{array}$ \\
\hline Microbiological response at the subject level \\
\hline $\begin{array}{l}\text { Eradication (documented or } \\
\text { presumed) }\end{array}$ & $\begin{array}{l}\text { None of the baseline pathogens were present in repeat intra-abdominal cultures from the original site of infection } \\
\text { taken during the study or a clinical response of cure precluded the necessity of a repeat intra-abdominal culture. }\end{array}$ \\
\hline $\begin{array}{l}\text { Persistence (documented or } \\
\text { presumed) }\end{array}$ & $\begin{array}{l}\text { Documented: any baseline intra-abdominal pathogen was present in the cultures obtained from the original site of the } \\
\text { intra-abdominal abscess, peritonitis, or surgical wound infection during the study; presumed: repeat microbiological } \\
\text { data were not obtained for a subject with a clinical response of failure. }\end{array}$ \\
\hline Superinfection & $\begin{array}{l}\text { Emergence of a new pathogen during therapy, at the site of infection with emergence or worsening of clinical signs } \\
\text { and symptoms of infection. }\end{array}$ \\
\hline Indeterminate & $\begin{array}{l}\text { Subjects who were lost to follow-up (failure to have an assigned clinical response); or who died within } 2 \text { days after } \\
\text { the first dose of investigational product for any reason; or who died after } 2 \text { days but prior to the TOC assessment } \\
\text { because of non-infection-related reasons (as judged by the investigator); or no baseline pathogens identified. }\end{array}$ \\
\hline
\end{tabular}

Abbreviations: EOT, end of treatment; TOC, test-of-cure; AE, adverse events.

subjects who had clinical evidence of a cIAI, as defined in the inclusion criteria, completed the TOC assessment with a response of cure or failure, and had no major protocol violations that would have an impact on the efficacy evaluation. The ME population included all CE subjects who had $\geq 1$ baseline isolate from the IAI site that was susceptible to both study drugs and a microbiological response of eradication, persistence, or superinfection. The clinical modified intent-to-treat (c-mITT) population included all mITT subjects who had clinical evidence of a cIAI as defined in the inclusion criteria. The microbiological modified intentto-treat (m-mITT) population included all c-mITT subjects 
who had one or more baseline isolates. The safety analysis set included all subjects who received $\geq 1$ dose of study drug.

\section{Statistical analysis}

Two-step comparison of the cure rate between treatment groups was conducted for the primary end point. Step 1 tested the primary hypothesis test of non-inferiority of tigecycline to imipenem/cilastatin. Non-inferiority was concluded if the lower limit of the two-sided 95\% CI (corrected for continuity with normal distribution approximation) $)^{17}$ of treatment difference in cure rate for the CE population was $>-15 \%$. With non-inferiority concluded, the superiority of tigecycline to imipenem/cilastatin was tested for the CE and mITT populations. Superiority was concluded if the lower limit of the two-sided $95 \%$ CI was $>0$ for both CE and mITT populations. The $95 \%$ CIs for within-treatment group response rates were calculated using Clopper and Pearson's method; ${ }^{18}$ for the one-sided and two-sided tests, $P$-values were at the significance level of 0.025 and 0.05 , respectively.

The secondary end points were analyzed similarly. Additional analyses for the clinical response based on the mITT, c-mITT, and m-mITT populations were also conducted. Clinical and microbiological responses by species or types of baseline isolates at both the TOC assessment and at the EOT were summarized for ME and m-mITT subjects. The $95 \%$ CIs of the within-group response rates were constructed using the method of Clopper and Pearson. ${ }^{18}$ Subgroup analyses were conducted, including: 1) clinical responses at the TOC assessment for the ME and m-mITT populations by bacteremia status, 2) clinical responses at the TOC assessment for the $\mathrm{CE}$ and c-mITT populations by primary clinical diagnosis, and 3) clinical and microbiological responses at the TOC and EOT assessments for the ME and m-mITT populations by infection type. The 95\% CIs for between-group comparison were calculated based on the Wilson score method corrected for continuity. ${ }^{19-21}$

The demographic parameters and baseline characteristics were summarized. All safety summaries utilized the safety analysis set.

\section{Results \\ Patients}

Of 470 subjects randomized, 235 were randomized to each group (Figure 1). The primary efficacy population (ie, CE population) included $89.2 \%$ subjects in the tigecycline and $88.7 \%$ subjects in the imipenem/cilastatin group.

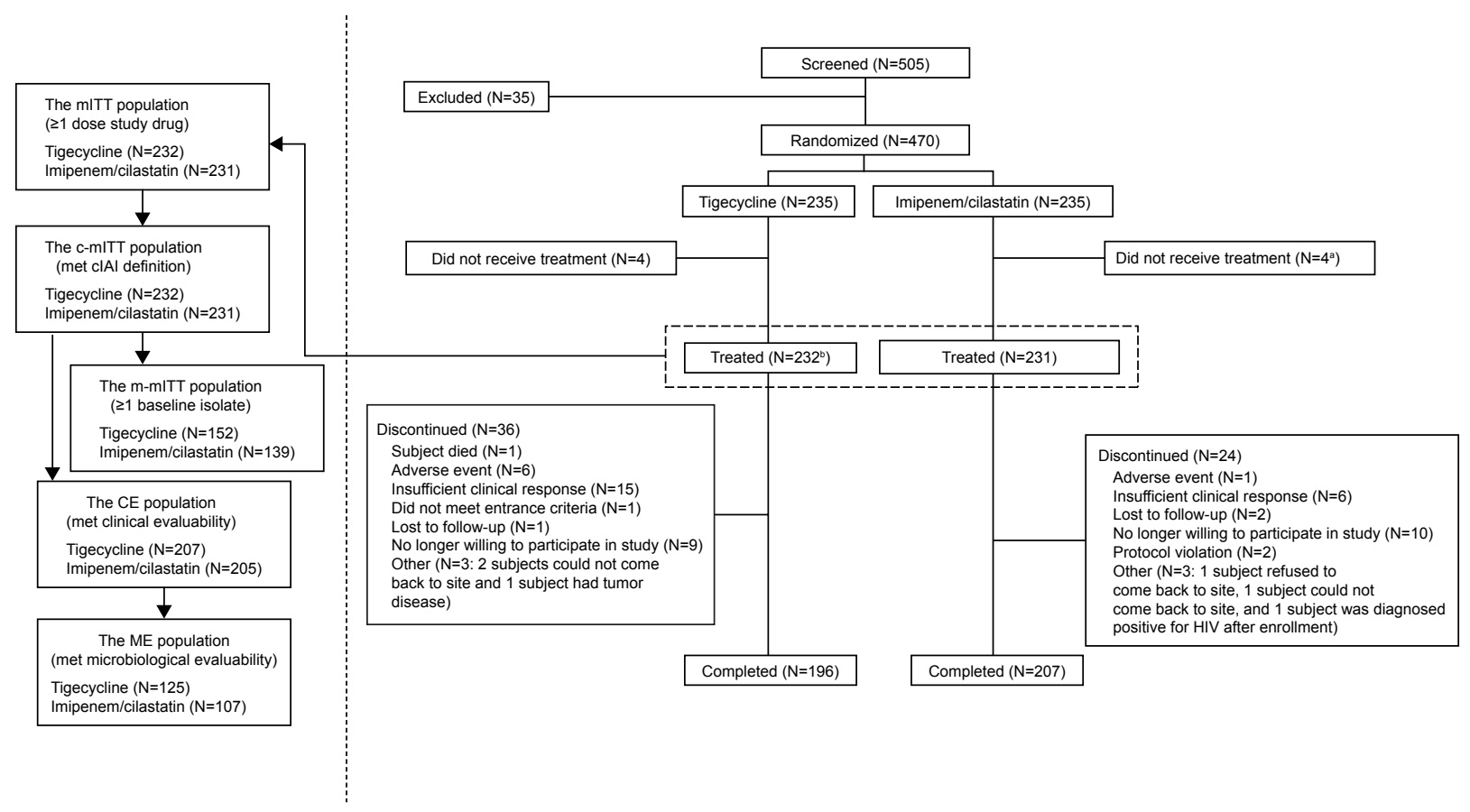

Figure I Patient disposition.

Notes: a One subject was randomized to imipenem/cilastatin group but received tigecycline throughout the treatment period. This subject was reported and analyzed under the tigecycline group for all efficacy and safety analyses and was excluded from the CE and ME populations. A sensitivity analysis to the primary end point of clinical response at the TOC assessment was conducted for the mITT population with this subject included in the imipenem/cilastatin group and the conclusions did not change. The procedures performed at TOC included: vital signs, assessment of clinical signs and symptoms of infection, urine or serum pregnancy test, urinalysis, hematology, serum chemistries, investigator assessment of clinical response, calculated creatinine clearance, microbiological determinations as in blood/intra-abdominal cultures, study drug administration, prior/concomitant medications/treatments, hospitalization information, and collection of adverse events. 'lncluding the subject described in the above footnote.

Abbreviations: CE, clinically evaluable; clAI, complicated intra-abdominal infection; c-mITT, clinical modified intent-to-treat; HIV, human immunodeficiency virus; ME, microbiologically evaluable; mITT, modified intent-to-treat; m-mITT, microbiological modified intent-to-treat; TOC, test-of-cure. 
Sixty subjects withdrew from the study; the most common reason was "insufficient clinical response" (6.5\%) for tigecycline and "no longer willing to participate in study" (4.3\%) for imipenem/cilastatin group (Figure 1).

The demographic characteristics were similar for the mITT (data not shown) and the CE population (Table 2). The most common ( $70 \%$ in each group) primary diagnosis was complicated appendicitis, and peritonitis due to perforation of small intestine was the second most common $(\sim 10 \%$ in each group). Other common primary diagnoses included perforated stomach, complicated cholecystitis, and peritonitis due to perforation of large intestine (Table 2).
All treated subjects received 80\%-120\% (except one tigecycline-treated subject who received $75 \%$ ) of the planned number of doses.

\section{Efficacy results}

\section{Primary end point: clinical response at the TOC} assessment

Among CE subjects, the cure rate was 89.9\% (186/207) for tigecycline- vs $96.6 \%$ (198/205) for imipenem/ cilastatin-treated subjects, and the difference in cure rate (tigecycline minus imipenem/cilastatin) was $-6.7 \%(95 \%$ CI: $-12.0 \%,-1.4 \%)$. Since the lower bound of the twosided $95 \%$ CI was $>-15 \%$, tigecycline was non-inferior

Table 2 Demographics and baseline characteristics of the CE population

\begin{tabular}{|c|c|c|}
\hline \multirow[t]{2}{*}{ Characteristic } & Tigecycline & Imipenem/cilastatin \\
\hline & $\mathbf{N}=\mathbf{2 0 7}$ & $\mathbf{N}=\mathbf{2 0 5}$ \\
\hline \multicolumn{3}{|l|}{ Age (years) } \\
\hline Mean (SD) & $47.3(17.7)$ & $48.7(17.4)$ \\
\hline Range & $18-85$ & $19-85$ \\
\hline \multicolumn{3}{|l|}{ Sex, $n$} \\
\hline Male & 132 & 133 \\
\hline Female & 75 & 72 \\
\hline \multicolumn{3}{|l|}{ Race, $\mathbf{n}$} \\
\hline Asian & 207 & 205 \\
\hline \multicolumn{3}{|l|}{ Weight (kg) } \\
\hline Mean (SD) & $61.7(12.1)$ & $62.0(11.4)$ \\
\hline Range & $40.0-130.0$ & $40.0-105.0$ \\
\hline \multicolumn{3}{|l|}{ Body mass index $\left(\mathrm{kg} / \mathrm{m}^{2}\right)$} \\
\hline Mean (SD) & $22.4(3.6)$ & $22.5(3.4)$ \\
\hline Range & $16.3-38.0$ & I5.5-33.I \\
\hline \multicolumn{3}{|l|}{ Height (cm) } \\
\hline Mean (SD) & $165.8(8.1)$ & I $65.6(8.1)$ \\
\hline Range & $136.0-186.0$ & $143.0-185.0$ \\
\hline \multicolumn{3}{|l|}{ Duration since diagnosis (days) } \\
\hline Mean (SD) & $1.2(0.53)$ & $1.3(1.39)$ \\
\hline Range & $0-5$ & $0-12$ \\
\hline \multicolumn{3}{|l|}{ APACHE II score } \\
\hline Mean (SD) & $5.2(3.38)$ & $5.4(3.38)$ \\
\hline Range & $0-15$ & $0-15$ \\
\hline \multicolumn{3}{|l|}{ APACHE II score categories, n (\%) } \\
\hline$\leq 15$ & $207(100.0)$ & $205(100.0)$ \\
\hline$>15$ & 0 & 0 \\
\hline \multicolumn{3}{|l|}{ Primary diagnosis, $\mathrm{n}(\%)$} \\
\hline Complicated appendicitis & $145(70.0)$ & $139(67.8)$ \\
\hline Peritonitis due to perforation of small intestine & $21(10.1)$ & $21(10.2)$ \\
\hline Perforated stomach & $18(8.7)$ & $18(8.8)$ \\
\hline Complicated cholecystitis & $10(4.8)$ & $13(6.3)$ \\
\hline Peritonitis due to perforation of large intestine ${ }^{a}$ & $4(1.9)$ & $5(2.4)$ \\
\hline Liver abscess & $3(1.4)$ & $4(2.0)$ \\
\hline Peritonitis $^{\mathrm{b}}$ & $I(0.5)$ & $4(2.0)$ \\
\hline Intra-abdominal abscess & $3(1.4)$ & $\mathrm{I}(0.5)$ \\
\hline Complicated diverticulitis & $2(1.0)$ & $0(0.0)$ \\
\hline
\end{tabular}

Notes: "Peritonitis due to perforation of large intestine due to diverticulitis" was included in this category; bincluded post-traumatic peritonitis. Abbreviations: APACHE, Acute Physiological and Chronic Health Evaluation; CE, clinically evaluable. 
Table 3 Clinical response at the test-of-cure assessment

\begin{tabular}{|c|c|c|c|c|c|c|c|c|}
\hline \multirow[t]{2}{*}{ Response } & \multicolumn{2}{|c|}{ Tigecycline $50 \mathrm{mg}$} & \multicolumn{2}{|c|}{ Imipenem/cilastatin } & \multicolumn{4}{|c|}{$\begin{array}{l}\text { Difference (tigecycline minus imipenem/ } \\
\text { cilastatin) }^{a}\end{array}$} \\
\hline & $n / N$ & $\%(95 \% \mathrm{Cl})^{b}$ & $n / N$ & $\%(95 \% \mathrm{Cl})^{\mathrm{b}}$ & $\%$ & 95\% Cl (\%) & $P$-value ${ }^{c}$ & $P$-value ${ }^{d}$ \\
\hline \multicolumn{9}{|l|}{ CE population } \\
\hline Cure & $186 / 207$ & $89.9(84.9,93.6)$ & $198 / 205$ & $96.6(93.1,98.6)$ & -6.7 & $(-12.0,-1.4)$ & 0.0008 & 0.0109 \\
\hline Failure & $21 / 207$ & $10.1(6.4,15.1)$ & $7 / 205$ & $3.4(1.4,6.9)$ & & & & \\
\hline \multicolumn{9}{|l|}{ mITT population } \\
\hline Cure & $192 / 232$ & $82.8(77.3,87.4)$ & $205 / 231$ & $88.7(83.9,92.5)$ & -6.0 & $(-12.8,0.8)$ & 0.0040 & 0.0861 \\
\hline Failure & $24 / 232$ & $10.3(6.7,15.0)$ & $8 / 231$ & $3.5(1.5,6.7)$ & & & & \\
\hline Indeterminate ${ }^{\mathrm{e}}$ & $16 / 232$ & $6.9(4.0,11.0)$ & $|8 / 23|$ & $7.8(4.7,12.0)$ & & & & \\
\hline \multicolumn{9}{|l|}{ ME population } \\
\hline Cure & $110 / 125$ & $88.0(81.0,93.1)$ & $102 / 107$ & $95.3(89.4,98.5)$ & -7.3 & $(-15.2,0.5)$ & 0.0277 & 0.0689 \\
\hline Failure & $15 / 125$ & $12.0(6.9,19.0)$ & $5 / 107$ & $4.7(1.5,10.6)$ & & & & \\
\hline
\end{tabular}

Notes: ${ }^{a} \mathrm{Cls}$ and $\mathrm{P}$-values for between-group comparison were calculated by the asymptotic method corrected for continuity with normal distribution approximation. ${ }^{b}$ Within-group $\mathrm{Cls}$ were calculated using the method of Clopper and Pearson. ${ }^{\mathrm{C}} P$-value for test for non-inferiority, one-sided at the level of 0.025 . ${ }^{\mathrm{d} P} \mathrm{\text {-value }}$ for test for superiority, two-sided at the level of 0.05 . eSubjects who were lost to follow-up (failure to have an outcome determination), or who died within 2 days after the first dose of investigational product for any reason, or who died after study Day 2 but prior to the test-of-cure assessment because of non-infection-related reasons (as judged by the investigator).

Abbreviations: CE, clinically evaluable; $n$, number of $\mathrm{CE}$, mITT, or ME subjects in each response category; $\mathrm{N}$, total number of subjects in CE, mITT, or ME populations; mITT, modified intent-to-treat; ME, microbiologically evaluable.

to imipenem/cilastatin with respect to clinical response at the TOC assessment (Table 3). In the mITT population, the difference in cure rate between treatment groups was $-6.0 \%$ $(-12.8 \%, 0.8 \%)$ (Table 3). Superiority of tigecycline over imipenem/cilastatin could not be demonstrated, as the lower bound of the two-sided $95 \% \mathrm{CI}$ was $<0$ for the $\mathrm{CE}$ and the mITT population. Superiority of imipenem/cilastatin over tigecycline was not demonstrated, since the upper bound of the two-sided $95 \%$ CI was $<0$ for the CE and $>0$ for the mITT population.

\section{Secondary end points}

For the ME population, the cure rate was $88.0 \%(110 / 125)$ for tigecycline- vs $95.3 \%$ (102/107) for imipenem/cilastatintreated subjects (difference $-7.3 \%[-15.2 \%, 0.5 \%]$ ), indicating that tigecycline did not meet the statistical criterion of non-inferiority to imipenem/cilastatin for the clinical response at TOC (Table 3). As cIAI is an invasive procedure and follow-up cultures at the site of infection are often not obtained, microbiological responses were most often presumed from clinical response. Therefore, the same results were observed for subject-level microbiological responses at TOC in the ME population.

\section{Other evaluations}

Results at EOT were generally consistent with those at TOC. With respect to clinical response at EOT for the $\mathrm{CE}$ and $\mathrm{ME}$ populations and subject-level microbiological response at EOT for the ME subjects, tigecycline was non-inferior to imipenem/cilastatin (data not shown).
In the ME population, 84 (67.2\%) tigecycline-treated and $81(75.7 \%)$ imipenem/cilastatin-treated subjects had monomicrobial infection; the eradication rate was $90.5 \%$ for tigecycline-treated and $93.8 \%$ for imipenem/cilastatintreated subjects at TOC (difference $-3.4 \%[-13.0 \%, 6.3 \%]$ ). For those with polymicrobial infections, the eradication rate was $82.9 \%$ for tigecycline-treated and $100.0 \%$ for imipenem/ cilastatin-treated subjects (difference $-17.1 \%$ [ $-32.6 \%$, $1.5 \%$ ]) (Table 4). For subjects infected by two pathogens, 30/36 tigecycline-treated subjects and 22/22 imipenem/ cilastatin-treated subjects were cured. Nine subjects (five tigecycline-treated and four imipenem/cilastatin-treated) were infected by three pathogens, and all were cured except one tigecycline-treated subject who was infected by Acinetobacter baumannii, Enterococcus avium, and Hafnia alvei (data not shown).

On a per-pathogen basis in the ME population, most pathogens $(90.6 \%$ for the tigecycline group and $96.3 \%$ for the imipenem/cilastatin group) were eradicated at the TOC assessment. The most commonly identified pathogen in the ME population was $E$. coli, for which the eradication rate was $92.1 \%$ for tigecycline and $96.4 \%$ for imipenem/ cilastatin groups. The second most common bacterial species was Klebsiella spp., for which 13 of $16(81.3 \%)$ isolates in the tigecycline group and 11 of $13(84.6 \%)$ isolates in the imipenem/cilastatin group were eradicated (Table 5).

Bacterial susceptibilities (tested by MIC) to tigecycline appeared to be consistent with clinical and microbiological responses. No subjects were identified with isolates that had decreased susceptibility ( $\geq 4$-fold increase in MIC from 
Table 4 Microbiological response at the subject level in the test-of-cure assessment for the ME population

\begin{tabular}{|c|c|c|c|c|c|c|c|c|}
\hline \multirow[t]{2}{*}{ Response } & \multicolumn{2}{|c|}{ Tigecycline 50 mg } & \multicolumn{2}{|c|}{ Imipenem/cilastatin } & \multicolumn{4}{|c|}{$\begin{array}{l}\text { Difference (tigecycline minus imipenem/ } \\
\text { cilastatin) }\end{array}$} \\
\hline & $n / N$ & $\%(95 \% \mathrm{Cl})^{\mathrm{a}}$ & $\mathbf{n} / \mathbf{N}$ & $\%(95 \% \mathrm{Cl})^{a}$ & $\%$ & 95\% Cl (\%) & $P$-value ${ }^{b}$ & $P$-value \\
\hline \multicolumn{9}{|l|}{ All subjects } \\
\hline Eradication & $110 / 125$ & $88.0(81.0,93.1)$ & $102 / 107$ & $95.3(89.4,98.5)$ & -7.3 & $(-15.2,0.5)^{\mathrm{d}}$ & $0.0277^{d}$ & $0.0689^{d}$ \\
\hline Persistence & $13 / 125$ & $10.4(5.7,17.1)$ & $5 / 107$ & $4.7(1.5,10.6)$ & & & & \\
\hline Superinfection ${ }^{e}$ & $2 / 125$ & $1.6(0.2,5.7)$ & $0 / 107$ & $0(0,3.4)$ & & & & \\
\hline \multicolumn{9}{|l|}{ Subjects with } \\
\hline \multicolumn{9}{|c|}{ monomicrobial infection } \\
\hline Eradication & $76 / 84$ & $90.5(82.1,95.8)$ & $76 / 81$ & $93.8(86.2,98.0)$ & -3.4 & $(-13.0,6.3)^{f}$ & & \\
\hline Persistence & $8 / 84$ & $9.5(4.2,17.9)$ & $5 / 81$ & $6.2(2.0,13.8)$ & & & & \\
\hline Superinfection ${ }^{e}$ & $0 / 84$ & $0.0(0.0,4.3)$ & $0 / 81$ & $0.0(0.0,4.5)$ & & & & \\
\hline \multicolumn{9}{|c|}{ Subjects with polymicrobial } \\
\hline \multicolumn{9}{|l|}{ infection $^{g}$} \\
\hline Eradication & $34 / 41$ & $82.9(67.9,92.8)$ & $26 / 26$ & $100.0(86.8,100.0)$ & -17.1 & $(-32.6,1.5)^{f}$ & & \\
\hline Persistence & $5 / 41$ & $12.2(4.1,26.2)$ & $0 / 26$ & $0.0(0.0,13.2)$ & & & & \\
\hline Superinfection $^{e}$ & $2 / 41$ & $4.9(0.6,16.5)$ & $0 / 26$ & $0.0(0.0,13.2)$ & & & & \\
\hline
\end{tabular}

Notes: aWithin-group Cls were calculated using the method of Clopper and Pearson. ${ }^{b} P$-value for test for non-inferiority, one-sided at the level of 0.025 . ${ }^{c}$-value for test for superiority, two-sided at the level of $0.05 .{ }^{\mathrm{d}} \mathrm{Cls}$ and $P$-values for between-group comparison were calculated by the asymptotic method corrected for continuity with normal distribution approximation. eSuperinfection was defined as emergence of a new pathogen during therapy, at the site of infection with emergence or worsening of clinical signs and symptoms of infection. ${ }^{\mathrm{f}} \mathrm{Cl}$ s were calculated based on the Wilson score method corrected for continuity. ${ }^{\mathrm{g} S u b j e c t s}$ with polymicrobial infections were infected by $\geq 2$ pathogens at baseline.

Abbreviations: ME, microbiologically evaluable; $n$, number of ME subjects with each microbiological response; N, number of ME subjects; $n$, number of ME subjects.

baseline) to tigecycline or imipenem/cilastatin during the study. The $\mathrm{MIC}_{50}$ and $\mathrm{MIC}_{90}$ values of tigecycline and imipenem against baseline isolates ( $\geq 5$ subjects in either group) in the ME population are presented in Table S2.
Further, clinical response was assessed in the ME population by baseline presence/absence of bacteremia and in the CE population by primary diagnosis. For those without bacteremia, the cure rate in tigecycline-treated vs

Table 5 Pathogen-level microbiological eradication rates by species or types of baseline isolates at the test-of-cure assessment for the ME population

\begin{tabular}{|c|c|c|c|c|}
\hline \multirow[t]{2}{*}{ Species or types of baseline isolates } & \multicolumn{2}{|c|}{ Tigecycline } & \multicolumn{2}{|c|}{ Imipenem/cilastatin } \\
\hline & $n / N$ & $\%(95 \% \mathrm{Cl})^{\mathrm{a}}$ & $\mathbf{n} / \mathbf{N}$ & $\%(95 \% \mathrm{Cl})^{\mathrm{a}}$ \\
\hline All pathogens ${ }^{b}$ & $154 / 170$ & $90.6(85.2,94.5)$ & $131 / 136$ & $96.3(91.6,98.8)$ \\
\hline Escherichia coli & $93 / 101$ & $92.1(85.0,96.5)$ & $81 / 84$ & $96.4(89.9,99.3)$ \\
\hline Klebsiella spp. ${ }^{c}$ & $13 / 16$ & $81.3(54.4,96.0)$ & $11 / 13$ & $84.6(54.6,98.1)$ \\
\hline Enterobacter spp. ${ }^{d}$ & $2 / 3$ & $66.7(9.4,99.2)$ & $2 / 2$ & $100.0(15.8,100.0)$ \\
\hline Other Enterobacteriaceae ${ }^{e}$ & $4 / 4$ & $100.0(39.8,100.0)$ & $1 / 1$ & $100.0(2.5,100.0)$ \\
\hline Other Gram-negative bacillus ${ }^{f}$ & $6 / 8$ & $75.0(34.9,96.8)$ & $6 / 6$ & $100.0(54.1,100.0)$ \\
\hline Enterococcus spp. ${ }^{\mathrm{g}}$ & $11 / 11$ & $100.0(71.5,100.0)$ & $4 / 4$ & $100.0(39.8,100.0)$ \\
\hline Staphylococcus aureus & $2 / 2$ & $100.0(15.8,100.0)$ & NA & NA \\
\hline Coagulase-negative staphylococci (CoNS) ${ }^{\mathrm{h}}$ & $3 / 3$ & $100.0(29.2,100.0)$ & $10 / 10$ & $100.0(69.2,100.0)$ \\
\hline Streptococcus agalactiae & NA & NA & $\mathrm{I} / \mathrm{I}$ & $100.0(2.5,100.0)$ \\
\hline Viridans group streptococci ${ }^{i}$ & $9 / 10$ & $90.0(55.5,99.7)$ & $11 / 11$ & $100.0(71.5,100.0)$ \\
\hline Other Gram-positive cocci & $1 / 2$ & $50.0(1.3,98.7)$ & NA & NA \\
\hline Bacteroides fragilis & $4 / 4$ & $100.0(39.8,100.0)$ & $\mathrm{I} / \mathrm{I}$ & $100.0(2.5,100.0)$ \\
\hline Other Bacteroides ${ }^{k}$ & $6 / 6$ & $100.0(54.1,100.0)$ & $2 / 2$ & $100.0(15.8,100.0)$ \\
\hline Clostridium perfringens & NA & NA & $\mathrm{I} / \mathrm{I}$ & $100.0(2.5,100.0)$ \\
\hline
\end{tabular}

Notes: A subject could have been counted more than once if multiple unique pathogens were isolated from this subject. Excluded Candida tropicalis and Curtobacterium. ${ }^{\mathrm{C} C l}$ were calculated using the method of Clopper and Pearson. 'For all pathogens, $\mathrm{N}$, number of pathogens isolated from all subjects; $\mathrm{n}$, number of pathogens isolated from all subjects that were eradicated. Included Klebsiella pneumoniae and Klebsiella oxytoca. Included Enterobacter aerogenes and Enterobacter cloacae. Included Proteus mirabilis, Proteus vulgaris group/Proteus penneri group, Plesiomonas shigelloides, and Hafnia alvei. flncluded Pseudomonas aeruginosa, Pseudomonas mendocina, Acinetobacter baumannii, Aeromonas hydrophila, Aeromonas salmonicida, and Comamonas testosteroni. IIncluded Enterococcus faecium and Enterococcus avium. 'Included Staphylococcus epidermidis, Staphylococcus hominis, Staphylococcus saprophyticus, and Staphylococcus xylosus. Included Streptococcus alactolyticus, Streptococcus anginosus, Streptococcus constellatus, Streptococcus gordonii,

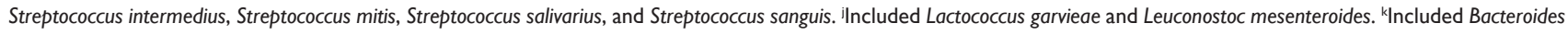
ovatus/Bacteroides thetaiotaomicron, Bacteroides splanchnicus, Bacteroides stercoris, and Bacteroides thetaiotaomicron.

Abbreviations: ME, microbiologically evaluable; $n$, number of pathogens isolated from all subjects that were eradicated for each species or type of bacteria; $\mathrm{N}$, number of pathogens isolated from all subjects for each species or type of bacteria; NA, not applicable; TOC, test-of-cure. 
imipenem/cilastatin-treated subjects was $86.4 \%(95 / 110)$ vs 95.8\% (91/95) (difference $-9.4 \%$ [-18.1\%, $-0.6 \%]$ ); for subjects with bacteremia, the cure rate was $100 \%(15 / 15)$ vs $91.7 \%$ (11/12) (difference 8.3\% [-18.2\%, 40.2\%]). The majority of subjects in the CE population (145 tigecyclinetreated and 139 imipenem/cilastatin-treated) were diagnosed with complicated appendicitis, among whom the clinical response in the tigecycline group was 135/145 (93.1\%) and 138/139 (99.3\%) in the imipenem/cilastatin group (difference $-6.2 \%[-12.0 \%,-1.1 \%]$ ) (data not shown).

\section{Safety evaluation}

The DMC reviewed the SAEs report approximately every half year and safety results in two additional safety data review meetings and recommended "continue the trial as designed". The safety analysis set included 463 subjects who received $\geq 1$ dose of tigecycline or imipenem/cilastatin (Figure 1). The mean treatment duration was 7.5 and 7.6 days for tigecycline- and imipenem/cilastatin-treated subjects, respectively.

\section{AEs and SAEs}

The incidence of all-causality and treatment-related AEs was higher in tigecycline-treated (56.5\% and $22.8 \%$ ) than in imipenem/cilastatin-treated subjects (46.8\% and $12.6 \%)$ (Table 6). Excluding AEs coded as "drug ineffective", the incidence of all-causality and treatment-related AEs was $54.3 \%$ and $18.5 \%$ with tigecycline vs $45.9 \%$ and $11.7 \%$ with imipenem/cilastatin, respectively.

The most frequent all-causality AEs in tigecyclinetreated subjects were nausea, drug ineffective, postoperative wound infection, vomiting, and pyrexia; the most frequent treatment-related AE was drug ineffective. None were unanticipated (Table 7).

The incidences of all-causality and treatment-related SAEs were both higher in tigecycline-treated than imipenem/ cilastatin-treated subjects (Table 6). The most frequent all-causality SAEs were drug ineffective (tigecycline: 21 [9.1\%]; imipenem/cilastatin: 9 [3.9\%]) and postoperative wound infection (7 [3.0\%] and 2 [0.9\%], respectively). Drug ineffective was also the most frequent treatment-related SAE (18 [7.8\%] and 6 [2.6\%], respectively). Excluding AEs coded as "drug ineffective", the incidences of all-causality and treatment-related SAEs were $6.9 \%$ and $1.3 \%$ with tigecycline vs $2.6 \%$ and $0.4 \%$ with imipenem/cilastatin, respectively.

The majority of AEs were mild in severity (tigecycline: 214/296, imipenem/cilastatin: 208/253). In tigecycline-treated subjects, 29 AEs were considered to be severe, including drug ineffective; lung infection; postoperative wound infection; atrial fibrillation; dyspnea; increased gamma-glutamyltransferase, alanine aminotransferase, and aspartate aminotransferase levels; abdominal infection; vomiting; sudden death; and hepatic failure. In imipenem/cilastatin-treated subjects, nine AEs were considered severe including drug ineffective, abdominal pain, pyrexia, postoperative wound infection, and increased amylase level. Table S3 lists AEs of special interest.

Discontinuations from study treatment (permanent or temporary) due to treatment-related AEs (excluding drug ineffective) were low overall $(<1 \%)$ and similar in both treatment groups (data not shown).

\section{Death}

Seven deaths were reported (four in the tigecycline group and three in the imipenem/cilastatin group). One subject

Table 6 Summary of treatment-emergent adverse events in the safety analysis set

\begin{tabular}{|c|c|c|c|c|}
\hline \multirow[t]{3}{*}{ Adverse events } & \multicolumn{2}{|c|}{ Tigecycline 50 mg } & \multicolumn{2}{|c|}{ Imipenem/cilastatin } \\
\hline & \multicolumn{2}{|l|}{$(\mathbf{N}=\mathbf{2 3 2})$} & \multicolumn{2}{|l|}{$(\mathbf{N}=\mathbf{2 3} \mathrm{I})$} \\
\hline & All-causality & Treatment-related & All-causality & Treatment-related \\
\hline Subjects in safety analysis set ${ }^{\mathrm{a}}, \mathrm{n}$ & 232 & 232 & 231 & 231 \\
\hline Number of AEs & 296 & 88 & 253 & 70 \\
\hline Subjects with AEs, n (\%) & $|3|(56.5)$ & $53(22.8)$ & $108(46.8)$ & $29(12.6)$ \\
\hline Subjects with SAEs, n (\%) & $32(13.8)$ & $19(8.2)$ & $15(6.5)$ & $7(3.0)$ \\
\hline Subjects with severe AEs, n (\%) & $21(9.1)$ & $13(5.6)$ & $9(3.9)$ & $5(2.2)$ \\
\hline $\begin{array}{l}\text { Subjects with dose reduced or temporary } \\
\text { discontinuation due to } A E s, n(\%)\end{array}$ & I $(0.4)$ & 0 & I (0.4) & I $(0.4)$ \\
\hline Subjects discontinued from study due to AEs, $n(\%)$ & $22(9.5)$ & $13(5.6)$ & $7(3.0)$ & $6(2.6)$ \\
\hline $\begin{array}{l}\text { Subjects permanently discontinued from study } \\
\text { treatment due to AEs, } n(\%)\end{array}$ & $20(8.6)$ & $14(6.0)$ & $2(0.9)$ & $2(0.9)$ \\
\hline
\end{tabular}

Notes: ancluded all data collected in the clinical database since the first dose of study drug. Per the protocol, SAEs occurring from the first dose of study treatment through last subject visit were required to be reported in the clinical database. AEs for one subject (imipenem/cilastatin group), which were reported after the database release, were not included in this table.

Abbreviations: $\mathrm{AE}$, adverse event; $\mathrm{N}$, total number of subjects; $\mathrm{SAE}$, serious $\mathrm{AE} ; \mathrm{n}$, number of subjects in each category. 
Table 7 Treatment-emergent adverse events in $\geq 2 \%$ of subjects in either treatment group in the safety analysis set

\begin{tabular}{|c|c|c|c|c|}
\hline \multirow[t]{3}{*}{ System organ class preferred term } & \multicolumn{2}{|l|}{ Tigecycline } & \multicolumn{2}{|c|}{ Imipenem/cilastatin } \\
\hline & \multicolumn{2}{|l|}{$(\mathrm{N}=\mathbf{2 3 2})$} & \multicolumn{2}{|l|}{$(\mathbf{N}=\mathbf{2 3} \mathrm{I})$} \\
\hline & All-causality & Treatment-related & All-causality & Treatment-related \\
\hline \multicolumn{5}{|l|}{ Clinical findings } \\
\hline \multicolumn{5}{|l|}{ Gastrointestinal disorders } \\
\hline Abdominal distension & $8(3.4)$ & 0 & $5(2.2)$ & 0 \\
\hline Diarrhea & II (4.7) & $4(1.7)$ & $7(3.0)$ & $2(0.9)$ \\
\hline Nausea & $24(10.3)$ & $16(6.9)$ & $10(4.3)$ & $5(2.2)$ \\
\hline Vomiting & $19(8.2)$ & $12(5.2)$ & $10(4.3)$ & $4(1.7)$ \\
\hline \multicolumn{5}{|c|}{ General disorders and administration site conditions } \\
\hline Drug ineffective & $21(9.1)$ & $18(7.8)$ & $9(3.9)$ & $6(2.6)$ \\
\hline Pyrexia & $18(7.8)$ & $\mathrm{I}(0.4)$ & $19(8.2)$ & $2(0.9)$ \\
\hline \multicolumn{5}{|l|}{ Infections and infestations } \\
\hline Lung infection & $7(3.0)$ & $\mathrm{I}(0.4)$ & $2(0.9)$ & 0 \\
\hline Postoperative wound infection & $21(9.1)$ & $3(1.3)$ & $3(1.3)$ & $\mathrm{I}(0.4)$ \\
\hline \multicolumn{5}{|l|}{ Metabolism and nutrition disorders } \\
\hline Hyperproteinemia & $4(1.7)$ & 0 & $9(3.9)$ & 0 \\
\hline \multicolumn{5}{|l|}{ Nervous system disorders } \\
\hline Dizziness & $3(1.3)$ & 0 & $5(2.2)$ & $3(1.3)$ \\
\hline \multicolumn{5}{|c|}{ Respiratory, thoracic, and mediastinal disorders } \\
\hline Cough & $5(2.2)$ & 0 & $9(3.9)$ & 0 \\
\hline Productive cough & $5(2.2)$ & 0 & $\mathrm{I}(0.4)$ & 0 \\
\hline \multicolumn{5}{|l|}{ Investigations } \\
\hline Alanine aminotransferase increased & $5(2.2)$ & $3(1.3)$ & $10(4.3)$ & $8(3.5)$ \\
\hline Aspartate aminotransferase increased & $4(1.7)$ & $3(1.3)$ & $10(4.3)$ & $8(3.5)$ \\
\hline Blood albumin decreased & $6(2.6)$ & 0 & $6(2.6)$ & $\mathrm{I}(0.4)$ \\
\hline Blood potassium decreased & $2(0.9)$ & 0 & $5(2.2)$ & $\mathrm{I}(0.4)$ \\
\hline Urine ketone body present & 0 & 0 & $6(2.6)$ & $2(0.9)$ \\
\hline Urobilinogen urine increased & 0 & 0 & $5(2.2)$ & $4(1.7)$ \\
\hline
\end{tabular}

Notes: Values are $\mathrm{n}(\%)$. AEs for one subject (imipenem/cilastatin group), which were reported after the database release, were not included in this table. Abbreviations: $\mathrm{AE}$, adverse event; $\mathrm{N}$, total number of subjects; $n$, number of subjects in each category.

died during the study within 1 day of the last treatment with tigecycline; this was a sudden death and not considered related to tigecycline. One subject died 38 days after the last tigecycline treatment, for whom two SAEs (drug ineffective and lung infection, both considered possibly related to tigecycline) with an outcome of death were reported. For the remaining deaths, one subject was randomized to the imipenem/cilastatin group but received no treatment, two were in the tigecycline group (died on Days 94 and 183), and two were in the imipenem/cilastatin group (died on Days 71 and 106).

\section{Laboratory test abnormalities}

Regardless of baseline values, 209/230 (91\%) tigecyclinetreated and 214/224 (96\%) imipenem/cilastatin-treated subjects had $\geq 1$ abnormal laboratory test result while on therapy or from EOT to TOC; the incidences of common (in $\geq 10 \%$ of subjects in either group) hematology and chemistry abnormalities are shown in Table S4.

\section{Discussion}

This Phase IV, multicenter, randomized, double-blind study has demonstrated that tigecycline is efficacious and generally well-tolerated in the treatment of hospitalized Chinese adult patients with cIAI. According to pre-specified statistical criteria, tigecycline was non-inferior to imipenem/ cilastatin with respect to clinical response at the TOC assessment for all CE subjects and all c-mITT/mITT subjects; superiority of tigecycline over imipenem/cilastatin or superiority of imipenem/cilastatin over tigecycline could not be demonstrated.

The cure rates in this study were consistent with other tigecycline cIAI trials, ${ }^{11,13,14,22}$ in particular a Phase III, multicenter, open-label study carried out in China between 2005 and 2006, in which clinical cure rates for tigecycline and imipenem/cilastatin at the TOC visit were $87.0 \%$ and $95.4 \%$, respectively, for the $\mathrm{CE}$ population. ${ }^{14}$ In the current study, the cure rate for tigecycline ranged from $82.8 \%$ to $89.9 \%$ for mITT, ME, and CE populations at TOC, indicating that 
tigecycline remained efficacious in treating cIAI in hospitalized patients in China.

The current study population included cIAI patients with monomicrobial or polymicrobial infections. Subgroup analysis showed that clinical response rates for tigecycline were generally similar to those for imipenem/cilastatin in subjects with monomicrobial infection and numerically lower in subjects with polymicrobial infection. However, the number of subjects assessed for polymicrobial infections was small, and there were no trends related to any single pathogen to explain this latter difference. Of note, the cure rate of tigecycline was lower for subjects with polymicrobial vs monomicrobial infection, a previously reported observation. . $^{11,14,23}$

Primary diagnosis categories for subjects in the current study included complicated appendicitis $(70 \%)$, peritonitis, peritonitis due to perforation of small or large intestine, diverticulitis, cholecystitis, intra-abdominal abscess, liver abscess, and perforated stomach. Efficacy of tigecycline was demonstrated across these diagnoses. In addition, as compared with imipenem/cilastatin, the clinical response rates for tigecycline were numerically higher in subjects with bacteremia, but the number of subjects with bacteremia was small.

In the ME population, tigecycline did not meet the statistical criterion of non-inferiority to imipenem/cilastatin for the clinical response at the TOC assessment or microbiological response at the subject level at the TOC assessment. However, the majority of the pathogens were eradicated for both treatment groups in the ME population. Bacterial susceptibilities to tigecycline appeared to be consistent with clinical responses and microbiological responses. No decreased susceptibility ( $\geq 4$-fold increase in MIC from baseline) to tigecycline or imipenem/cilastatin was identified. In this study, E. coli was the most commonly identified pathogen and remained sensitive to tigecycline and imipenem. Considering that multidrug resistant $E$. coli is prevalent in the Asia-Pacific region (13.7\% before 2010) and lately tigecycline-resistant $E$. coli isolates have been identified globally, ${ }^{24}$ the possibility of tigecycline-resistant E. coli emergence cannot be ruled out. Therefore, bacterial resistance to tigecycline should be monitored closely.

During the course of this study, no unanticipated AEs were reported. The higher occurrence of nausea and vomiting in the tigecycline group vs the imipenem/cilastatin group was consistent with the known safety profile of tigecycline. ${ }^{11,14,22}$ Incidence of treatment-related SAEs, excluding drug ineffective (considered an SAE in this study), was low and similar between the two groups (three subjects in the tigecycline and one subject in the imipenem/cilastatin group). Four deaths in the tigecycline group and three deaths in the imipenem/ cilastatin group were reported in this study. Although allcause mortality was noted to be higher in patients treated with tigecycline than comparators in a meta-analysis of clinical trials, ${ }^{10,15}$ further analyses have suggested that in cIAI trials tigecycline is not a significant factor for death. ${ }^{25}$

The revised Surgical Infection Society's guidelines for managing IAI recommend tigecycline for pathogendirected use for resistant Gram-negative bacteria other than Pseudomonas spp. in adult patients. ${ }^{26}$ The study was sufficiently powered to conduct formal statistical analysis for non-inferiority of the CE population (as the primary efficacy population), but not the ME population, which may explain why the statistical criterion of non-inferiority to imipenem/ cilastatin for the clinical response at the TOC assessment for the ME population was not met.

In summary, tigecycline was non-inferior to imipenem/ cilastatin in treating hospitalized adult patients with cIAI in China; superiority of tigecycline over imipenem/cilastatin or superiority of imipenem/cilastatin over tigecycline could not be concluded. In addition, bacterial susceptibilities to tigecycline were consistent with clinical responses and microbiological responses. Further, the observed safety in this study was consistent with the known profile for tigecycline with no unanticipated safety findings.

\section{Acknowledgments}

The study results were submitted to ClinicalTrial.gov and are currently under review at the time of manuscript submission. Editorial support was provided by Shuang Li, $\mathrm{PhD}$, at Engage Scientific (Horsham, UK) and was funded by Pfizer.

This study was sponsored by Pfizer. Editorial support was funded by Pfizer. Upon request and subject to certain criteria, conditions, and exceptions (see https://www.pfizer. com/science/clinical-trials/trial-data-and-results for more information), Pfizer will provide access to individual deidentified participant data from Pfizer-sponsored global interventional clinical studies conducted for medicines, vaccines, and medical devices 1) for indications that have been approved in the US and/or EU or 2) in programs that have been terminated (ie, development for all indications has been discontinued). Pfizer will also consider requests for the protocol, data dictionary, and statistical analysis plan. Data may be requested from Pfizer trials 24 months after study completion. The de-identified participant data will be made 
available to researchers whose proposals meet the research criteria and other conditions, and for which an exception does not apply, via a secure portal. To gain access, data requestors must enter into a data access agreement with Pfizer.

\section{Study investigators}

Yongjie Zhao, Gang Chen, Ping Li, Jian Lei, Jiye Zhu, Dianrong Xiu, Chungen Xing, Yingyuan Zhang, Jufang Wu, Yijian Chen, Feizhou Huang, Lu Liang, Weilin Wang, Shuanghai Liu, Yongkuan Cao, Tianzheng Lou, Tienan Bi, Xiaoping Chen, Kailun Zhou, Weixing Shen, Hong Zhao, Zheng Zhou, Yongqiang Zhan, Mingyu Hu, Mingzhi Cai, Wei Li, Shoubai Li, Zhiqiang Hu, Qing Ni, Yamin Guo, Yuming Li, Guanbao Zhu, Yunlong Pan, Qian Chen, Xudong Wang, Tong Liu, Yuhong Wang, Guoyang Wu, Li Yu, Yuanzhou Shan, Taiping Zhang, Yong Yang, Naiqiang Cui, Nengwei Zhang, Lianchen Wang, Zhiming Wang, Zhaohui Du, Guangyi Wang, Bo Peng, Zhiwei Quan, Zhiyong Wu, Wenhui Lou, Lunan Yan, Qi Zheng, Xiaochun Ma, Jianwei Bi, Junmin Wei, Xiaohong Yu, Jixiang Wu and Zhongqing Chen. For detailed information of the study centers, see Table S5.

\section{Author contributions}

Yijian Chen, Yingyuan Zhang, Yongjie Zhao, Gang Chen, and Ping Li were study investigators and participated in study design, patient recruitment, acquisition of data, and/or analysis and interpretation of the findings. Demei Zhu was responsible for microbiological determination, study results analysis, and data interpretation. Ping Yan, M Anne Hickman, Margaret Tawadrous, and Michele Wible are employees of Pfizer; Lihong Xu and Xiajun Xu were employees of Pfizer; they participated in study design, analysis of data, and/or interpretation of the findings. Ping Yan and Michele Wible also provided statistical support. All authors contributed to data analysis, drafting and revising the article, gave final approval of the version to be published, and agree to be accountable for all aspects of the work.

\section{Disclosure}

No author received an honorarium related to the development of this manuscript. Yijian Chen, Demei Zhu, Yingyuan Zhang, Yongjie Zhao, Gang Chen, and Ping Li have nothing else to disclose. Lihong $\mathrm{Xu}$ and Xiajun $\mathrm{Xu}$ were employees of Pfizer. Ping Yan, M. Anne Hickman, Margaret Tawadrous, and Michele Wible are employees of Pfizer and may own company stock.

\section{References}

1. Solomkin JS, Mazuski JE, Bradley JS, et al. Diagnosis and management of complicated intra-abdominal infection in adults and children: guidelines by the Surgical Infection Society and the Infectious Diseases Society of America. Clin Infect Dis. 2010;50(2):133-164.

2. Sartelli M, Catena F, Ansaloni L, et al. Complicated intra-abdominal infections worldwide: the definitive data of the CIAOW Study. World J Emerg Surg. 2014;9:37.

3. Kurup A, Liau KH, Ren J, et al. Antibiotic management of complicated intra-abdominal infections in adults: The Asian perspective. Ann Med Surg. 2014;3(3):85-91.

4. Mikamo H, Yuasa A, Wada K, Crawford B, Sugimoto N. Optimal Treatment for Complicated Intra-abdominal Infections in the Era of Antibiotic Resistance: A Systematic Review and Meta-Analysis of the Efficacy and Safety of Combined Therapy With Metronidazole. Open Forum Infect Dis. 2016;3(3):ofw143.

5. Ouyang W, Xue H, Chen Y, et al. Clinical characteristics and antimicrobial patterns in complicated intra-abdominal infections: a 6-year epidemiological study in southern China. Int J Antimicrob Agents. 2016; 47(3):210-216.

6. Baquero F, Hsueh P-R, Paterson DL, et al. In vitro susceptibilities of aerobic and facultatively anaerobic gram-negative bacilli isolated from patients with intra-abdominal infections worldwide: 2005 results from Study for Monitoring Antimicrobial Resistance Trends (SMART). Surg Infect. 2009;10(2):99-104.

7. Hsueh PR. Study for Monitoring Antimicrobial Resistance Trends (SMART) in the Asia-Pacific region, 2002-2010. Int J Antimicrob Agents. 2012;40(Suppl):S1-S3.

8. Yang Q, Zhang H, Wang Y, et al. A 10 year surveillance for antimicrobial susceptibility of Escherichia coli and Klebsiella pneumoniae in community- and hospital-associated intra-abdominal infections in China. J Med Microbiol. 2013;62(Pt 9):1343-1349.

9. Jean SS, Hsueh PR; SMART Asia-Pacific Group. Distribution of ESBLs, AmpC $\beta$-lactamases and carbapenemases among Enterobacteriaceae isolates causing intra-abdominal and urinary tract infections in the AsiaPacific region during 2008-14: results from the Study for Monitoring Antimicrobial Resistance Trends (SMART). J Antimicrob Chemother. 2017;72(1):166-171.

10. Pfizer. Tygacil ${ }^{\circledR}$ U.S. Physician Prescribing Information. Available from: http://www.accessdata.fda.gov/drugsatfda_docs/label/2016/ 021821s0391bl.pdf. Accessed 29 July, 2018.

11. Fomin P, Beuran M, Gradauskas A, et al. Tigecycline is efficacious in the treatment of complicated intra-abdominal infections. Int J Surg. 2005;3(1):35-47.

12. Towfigh S, Pasternak J, Poirier A, Leister H, Babinchak T. A multicentre, open-label, randomized comparative study of tigecycline versus ceftriaxone sodium plus metronidazole for the treatment of hospitalized subjects with complicated intra-abdominal infections. Clin Microbiol Infect. 2010;16(8):1274-1281.

13. Qvist N, Warren B, Leister-Tebbe H, et al. Efficacy of tigecycline versus ceftriaxone plus metronidazole for the treatment of complicated intra-abdominal infections: results from a randomized, controlled trial. Surg Infect. 2012;13(2):102-109.

14. Chen Z, Wu J, Zhang Y, et al. Efficacy and safety of tigecycline monotherapy vs. imipenem/cilastatin in Chinese patients with complicated intra-abdominal infections: a randomized controlled trial. BMC Infect Dis. 2010;10:217.

15. Pfizer China. Tygacil ${ }^{\circledR}$ China Physician Prescribing Information. Available from: https:/www.pfizermedicalinformation.cn/OpenDocument. aspx?DocumentID=73c20343-2116-4078-910a-ab9fc4f6e6ea\&Type= PackageInserts. Accessed 29 July, 2018.

16. United States FDA Tygacil ${ }^{\circledR}$ (tigecycline) label information; 2013. Available from: http://www.accessdata.fda.gov/drugsatfda_docs/ label/2013/021821s026s0311bl.pdf. Accessed 29 July, 2018.

17. Fleiss JL. Statistical Methods for Rates and Proportions. 2nd ed. New York: John Wiley \& Sons; 1981:29-30. 
18. Clopper CJ, Pearson ES. The use of confidence or fiducial limits illustrated in the case of the binomial. Biometrika. 1934;26(4):404-413.

19. Newcombe RG. Interval estimation for the difference between independent proportions: comparison of eleven methods. Stat Med. 1998;17(8): 873-890.

20. Wilson EB. Probable inference, the law of succession, and statistical inference. J Am Stat Assoc. 1927;22(158):209-212.

21. Newcombe RG. Two-sided confidence intervals for the single proportion: comparison of seven methods. Stat Med. 1998;17(8): 857-872.

22. Oliva ME, Rekha A, Yellin A, et al. A multicenter trial of the efficacy and safety of tigecycline versus imipenem/cilastatin in patients with complicated intra-abdominal infections [Study ID Numbers: 3074A1301-WW; ClinicalTrials.gov Identifier: NCT00081744]. BMC Infect Dis. 2005;5:88
23. Babinchak T, Ellis-Grosse E, Dartois N, et al. The efficacy and safety of tigecycline for the treatment of complicated intra-abdominal infections: analysis of pooled clinical trial data. Clin Infect Dis. 2005;41(Suppl 5): S354-S367.

24. Giammanco A, Calà C, Fasciana T, Dowzicky MJ. Global Assessment of the Activity of Tigecycline against Multidrug-Resistant Gram-Negative Pathogens between 2004 and 2014 as Part of the Tigecycline Evaluation and Surveillance Trial. mSphere. 2017;2(1):e00310-e00316.

25. Bassetti M, McGovern PC, Wenisch C, et al. Clinical response and mortality in tigecycline complicated intra-abdominal infection and complicated skin and soft-tissue infection trials. Int J Antimicrob Agents. 2015;46(3):346-350.

26. Mazuski JE, Tessier JM, May AK, et al. The Surgical Infection Society Revised Guidelines on the Management of Intra-Abdominal Infection. Surg Infect. 2017;18(1):1-76.

\section{Publish your work in this journal}

Therapeutics and Clinical Risk Management is an international, peerreviewed journal of clinical therapeutics and risk management, focusing on concise rapid reporting of clinical studies in all therapeutic areas outcomes, safety, and programs for the effective, safe, and sustained use of medicines. This journal is indexed on PubMed Central, CAS,
EMBase, Scopus and the Elsevier Bibliographic databases. The manuscript management system is completely online and includes a very quick and fair peer-review system, which is all easy to use. Visit http://www.dovepress.com/testimonials.php to read real quotes from published authors.

Submit your manuscript here: http://www.dovepress.com/therapeutics-and-clinical-risk-management-journal 